\title{
Modulare Förderspirale aus Kunststoff
}

\author{
Dipl.-Ing. Arndt Schumann, Prof. Dr.-Ing. Klaus Nendel, Dr.-Ing. Thomas Linke, \\ Dipl.-Ing. Sebastian Weise \\ Technische Universität Chemnitz \\ Institut Fördertechnik und Kunststoffe \\ Professur Fördertechnik
}

\begin{abstract}
Der Einsatz von Spiralförderanlagen oder Schneckenförderanlagen ohne Welle ist in der Industrie weit verbreitet. Durch die Rotation der Spirale in einem ruhenden Rohr oder Trog lassen sich Schüttgüter unterschiedlichster Konsistenz kontinuierlich über weite Strecken transportieren.
\end{abstract}

Derzeitige Spiralförderanlagen werden überwiegend aus Stahl hergestellt. Bei einigen Anwendungen ist der Einsatz dieses Werkstoffes jedoch nicht unproblematisch. Aggressive Schüttgüter wie salz- und säurehaltige Medien bewirken eine starke Korrosion der Stahlbauteile. Kurze Standzeiten sind die Folge.

Diese Problematik sowie die aufwendige Montage und Reparatur bestehender Systeme bildeten die Herausforderungen für die Neuentwicklung.

Eine Vielzahl thermoplastischer Kunststoffe ist beständig gegen aggressive Medien wie Salzlösungen und Säuren. Innerhalb des Förderprogramms PRO INNO II wurde eine Spirale aus Kunststoff entwickelt, die korrosionsempfindliche Stahlspiralen ersetzt. Die neue Förderspirale wird aus spritzgegossenen Modulen kostengünstig hergestellt. Die Gestaltung der Verbindungsstellen erfolgte in der Weise, dass jedes Modul die gleiche Geometrie aufweist. Somit ist nur ein Spritzgießwerkzeug zur Herstellung der Bauteile notwendig. Die Montage der Module erfolgt mit geringstem Platzbedarf und Aufwand vor Ort. Das Spritzgießverfahren ermöglicht in Abhängigkeit von den Schüttguteigenschaften die Herstellung der Bauteile aus verschiedenen verstärkten und unverstärkten Kunststoffen.

\section{$1 \quad$ Einleitung}

Der Einsatz von Spiralförderern, die auch als Schneckenförderer ohne Welle oder Wendelförderer bezeichnet werden, ist in vielen Industriezweigen nicht mehr wegzudenken. Durch die Rotation einer Spirale in einem ruhenden Rohr oder Trog lassen sich Materialien unterschiedlichster Konsistenz kontinuierlich sowohl horizontal als auch vertikal transportieren. Zu diesen Materialien gehören korn- und staubförmige Güter wie Kalk, Glas, Salz, Mehl, Holzspäne und -pellets, pastöse Massen wie Teig, faserförmige Stoffe sowie hochviskose Suspensionen. In Abhängigkeit vom Fördergut kommen verschiedene Spiralgeometrien, -querschnitte sowie Rohr- oder Trogformen zum Einsatz.

Förderspiralen bestehen derzeit aus Stahl und werden durch Kaltwalzen und Schweißen hergestellt. In einigen Industriezweigen (Bsp.: Kaliindustrie), in denen 
Säuren, Basen oder gelöste Salze in den Fördergütern enthalten sind, ist der Einsatz von Stahlspiralen jedoch äußerst problematisch. Die im Fördergut enthaltenen aggressiven und abrasiven Substanzen bewirken enormen Verschleiß des Stahls. Folglich kommt es zu kurzen Austausch- und Wartungsintervallen.

Zielstellung war es eine Spiralförderanlage zu entwickeln, die sich vor allem durch die Korrosions- und Verschleißbeständigkeit auszeichnet. Der Fokus lag dabei auf der Entwicklung einer Spirale aus Kunststoff, die in der Massenproduktion durch Spritzgießen hergestellt werden sollte. Diese Zielstellung wurde durch eine Modulbauweise realisiert, welche gleichzeitig einige Vorteile, wie den platzsparenden Transport, die Montage vor Ort sowie eine individuelle Gestaltung der Länge mit sich bringt. Bei der Entwicklung mussten einige konstruktive Schwierigkeiten überwunden werden. So sind die Bauteilgröße, die Wandstärke und die Gestaltung von der Spritgießherstellung abhängig. Des Weiteren erreichen selbst faserverstärkte Kunststoffe nicht die Festigkeiten und Steifigkeiten der Stahlwerkstoffe. Die Gestaltung der Verbindungsstellen war nicht trivial. Zum einen sollte eine einfache Montage und zum anderen eine hohe Verbindungsfestigkeit erreicht werden. Die Geometrie der Module trägt diesen Anforderungen Rechnung.

Die Entwicklung erfolgte im Rahmen des Förderprogramms PRO INNO II. Die beteiligten Industriepartner waren die KD Stahl- und Maschinenbau GmbH und die Albert Polenz GmbH \& Co.KG.

\section{Stand der Technik}

\subsection{Aufbau und Wirkungsweise von Spiralförderanlagen}

Spiralförderanlagen ermöglichen den geschlossenen Transport von Schüttgütern über Entfernungen von wenigen Metern bis zu 70 m [App11]. Die folgende Abbildung zeigt die Prinzipdarstellung eines Spiralförderers.

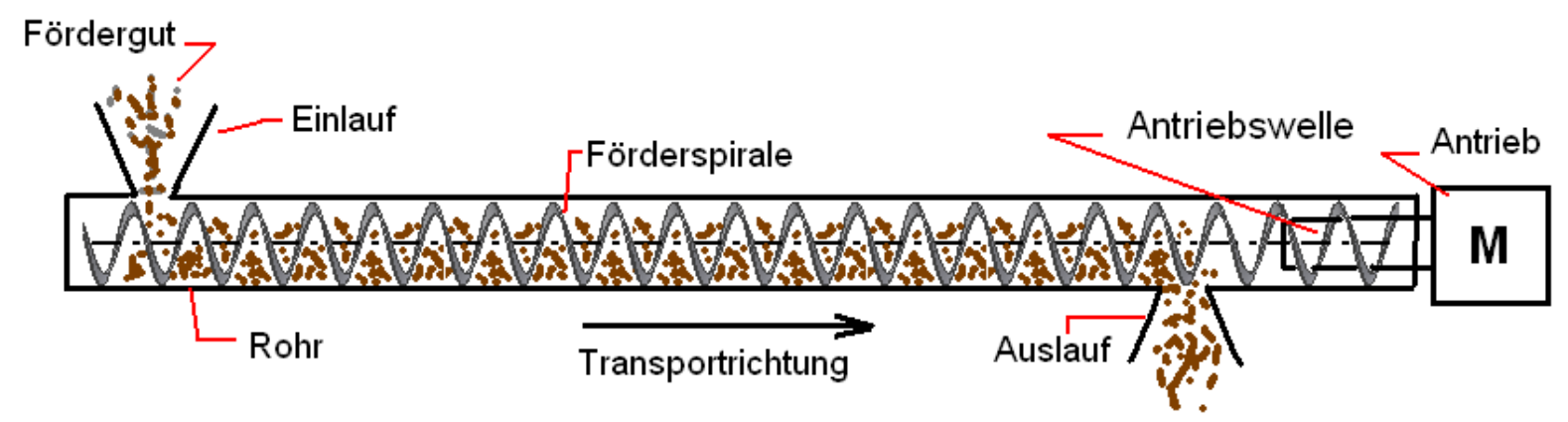

Abbildung 1: Prinzipdarstellung eines Spiralförderers

Derartige Anlagen sind für den waagerechten, steigenden bis vertikalen Betrieb geeignet. 
Es wird zwischen Fördersystemen mit biegeflexibler Spirale und Fördersystemen mit biegesteifer Spirale unterschieden. Durch biegeflexible Spiralen ist ein gekrümmter Verlauf der Förderanlage entsprechend der räumlichen Gegebenheiten möglich.

Neben dem Transport kommen Spiralfördersysteme zum Klassieren, beispielsweise von Abwässern, zur Anwendung. Ein weiterer Einsatzbereich ist das Mischen und Dosieren von Schüttgütern.

Die Geometrie der Spiralen weist charakteristische Merkmale auf. Zu nennen sind die Länge, der Außendurchmesser, der Innendurchmesser, die Blattstärke, die Blatthöhe, die Steigung, die Neigung des Querschnitts zur Längsachse sowie die Drehrichtung (Abbildung 2).

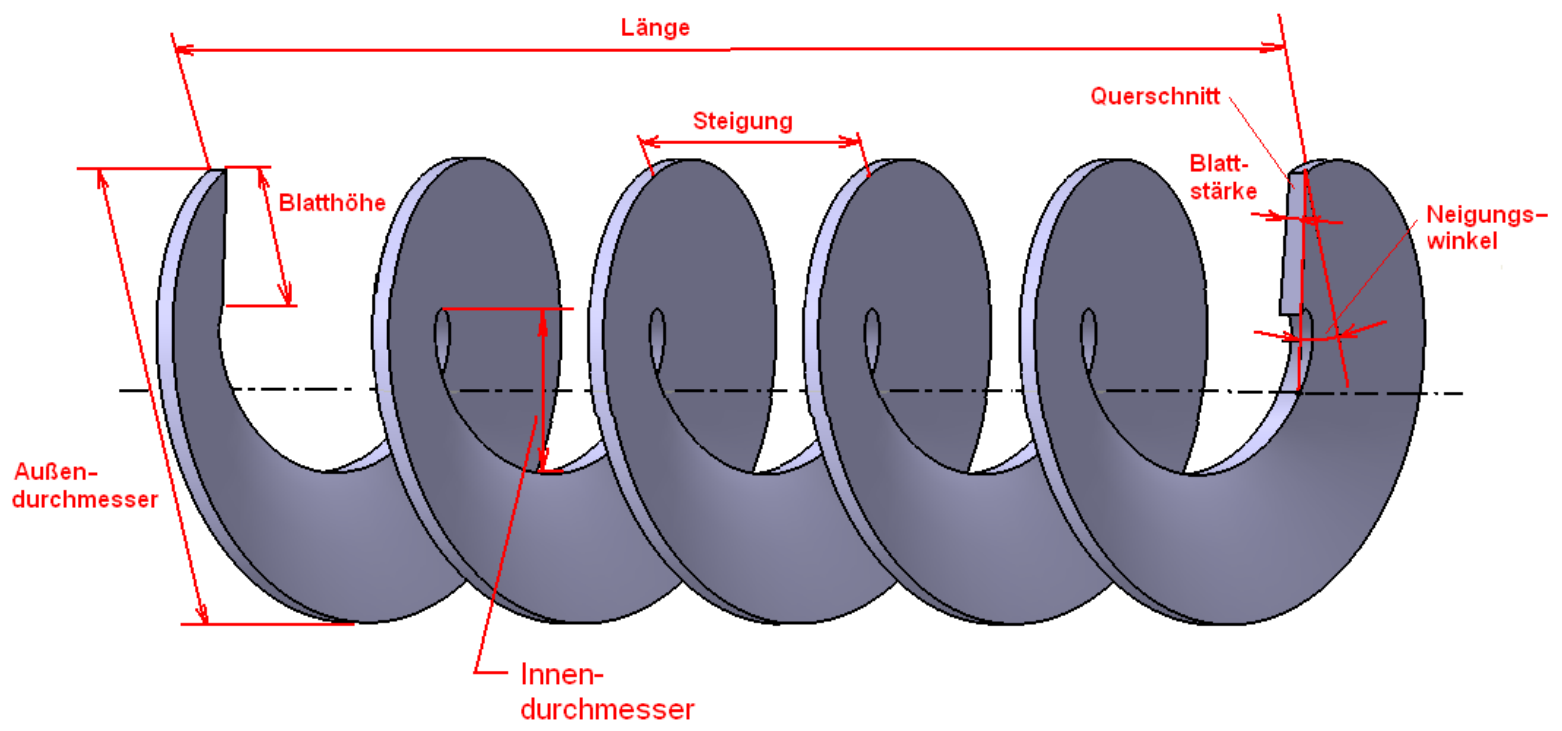

Abbildung 2: Geometrie einer Förderspirale

Neben dem Rechteckquerschnitt der Spirale sind auch andere Querschnittformen, wie beispielsweise kreisrunde und ovale, möglich. Der Außendurchmesser handelsüblicher Produkte [Ste11] reicht von wenigen Millimetern bis zu 600 Millimeter. Die Steigung entspricht meist dem Außendurchmesser. Modelle mit Blattstärke bis zu 20 Millimeter sind erhältlich.

Die Spirale wird durch den Trog geführt und ist einseitig über eine Welle mit dem Antrieb verbunden (Abbildung 1). Es existieren verschiedene Ausführungsformen des Troges. Zu nennen sind der Rohrtrog, der U-Trog, der scharfkantige U-Trog, der VTrog sowie der Doppeltrog (Abbildung 3). Meist sind die Tröge durch ein Blech nach oben verschlossen. 


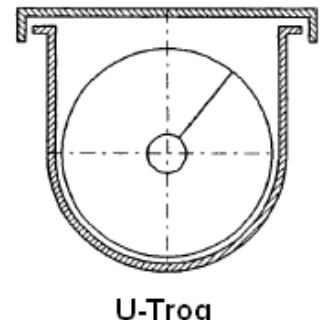

U-Trog

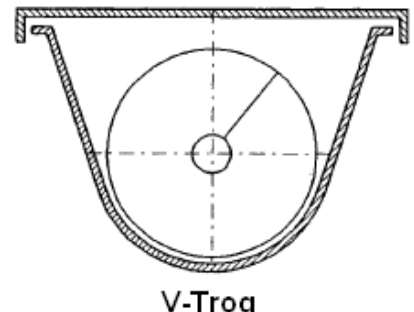

V-Trog

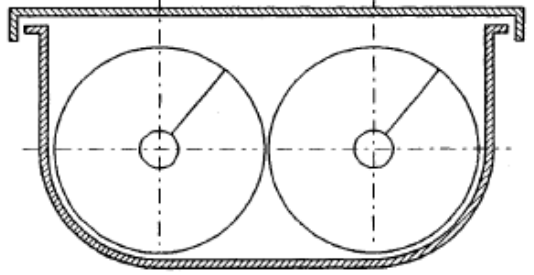

Doppeltrog

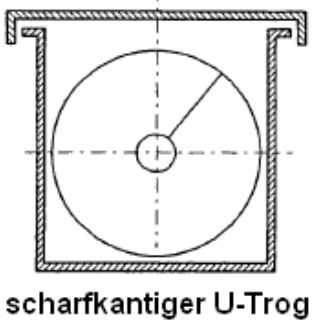

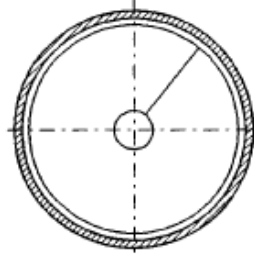

Rohrtrog

Abbildung 3: Überblick Trogformen [VDI93]

Die Zuführung des Schüttgutes in die Förderanlage wird über den Einlauf realisiert. Nach dem Transport über eine bestimmte Strecke erfolgt die Abgabe des Schüttgutes über den Auslauf (Abbildung 1). Es existieren auch Anlagen mit mehreren Einläufen und Ausläufen. Die Positionierung des Antriebes kann auslaufseitig oder einlaufseitig erfolgen. Befindet sich der Antrieb auf der Einlaufseite, spricht man von einem schiebenden Antrieb, anderenfalls von einem ziehenden Antrieb.

Sowohl in den Ausführungen des Spiralförderers als Transportorgan, als auch in der Funktion als Siebklassierer und technisches Mittel zum Dosieren wird das in den Trog gefüllte Schüttgut transportiert. Diese Funktion wird über die in Rotation versetzte Spirale in dem ruhenden Trog erreicht. Durch die Spiralflanke wird das Gut kontinuierlich voran geschoben. Dabei muss ein bestimmter Mindestreibungswiderstand zwischen Schüttgut und Trogwandung vorherrschen, der über dem Reibungswiderstand zwischen Schüttgut und Spiraloberfläche liegt, andernfalls dreht sich das zu transportierende Material um die Spiralachse. Im Idealfall bewegt sich das Fördergut bei einer Umdrehung der Spirale um den Betrag der Spiralsteigung in axialer Richtung. Umso schräger die Förderanlage im Raum installiert ist, desto geringer ist der Vorschub pro Umdrehung. Ab einem bestimmten Winkel muss sogar eine kritische Drehzahl überwunden werden, damit sich das Gut infolge der Fliehkräfte an die Trogwand böscht und somit eine bestimmte Reibkraft erreicht wird, die den Fördervorgang ermöglicht [DIN83] [VDI93].

\subsection{Einsatzbereiche und Schüttgüter}

Spiralförderanlagen kommen in vielen Industriebereichen zur Anwendung. Zu nennen sind in diesem Zusammenhang die Chemische Industrie, die Lebensmittelindustrie, die Bauindustrie, die Holzindustrie, Kläranlagen, Versatzanlagen, die Papierindustrie, Brauereien, die Textilindustrie, 
Kompostieranlagen, die Gemüseverarbeitung, die Obstverarbeitung, die Verarbeitung von Fisch und Meeresfrüchten, die Fleischverarbeitung, die Geflügelverarbeitung, die Zuckerindustrie und in Großküchen.

Die zu entwickelnde Spiralförderanlage soll vorerst in der Versatzanlagentechnik zur Anwendung kommen. Bei den dort zu fördernden Schüttgütern handelt es sich um industrielle und gewerbliche Abfälle wie mineralische Stäube, metallhaltige Stäube, Aschen, Granulate und Laugen. Diese Stoffe haben folgende chemische und physikalische Eigenschaften: salzhaltig, basisch, neutral, abrasiv, hygroskopisch, anbackend, Schüttdichte $=0,5<1,6 \mathrm{t} / \mathrm{m}^{3}$, Korngröße bis $30 \mathrm{~mm}$.

\subsection{Herstellung von Förderspiralen und Maßnahmen zur Erhöhung der Anlagenstandzeiten}

Die Fertigung von Förderspiralen ist durch folgende Arbeitsschritte gekennzeichnet: Aus Blechen werden zunächst Kreisringe mittels Wasserstrahlschneiden, Plasmaschneiden, Laserschneiden oder Stanzen ausgeschnitten. Anschließend erfolgt das Biegen der Kreisringe durch Kaltumformung entsprechend der Steigung der Spirale. Im Anschluss wird die Verbindung der Teile durch Schweißen hergestellt. Um eine optimale Funktionalität der Förderanlage zu erreichen, ist eine glatte Spiraloberfläche notwendig. Demzufolge müssen die Schweißnähte verschliffen werden. Nach diesen Arbeitsschritten ist die Antriebswelle an die Spirale zu schweißen. Abschließend wird die Spirale lackiert.

Bei der zweiten Fertigungsmethode werden Blechbänder oder Rundstahlstäbe in Form gewalzt. Es entstehen somit Spiralabschnitte, die entsprechend der vorgegebenen Länge zusammenzuschweißen bzw. zu kürzen sind.

Zur Erhöhung der Standzeit von Spiralförderanlagen werden verschiedene Maßnahmen angestrengt. Ziel dabei ist es, die Korrosions- und Verschleißbeständigkeit zu erhöhen. So kommen hochlegierte, nicht rostende oder gehärtete Stähle zum Einsatz. Des Weiteren werden spezielle Verfahren zur Beschichtung der Oberfläche mit Metallegierungen angewandt. Bei dem Metallspritzen wird schmelzflüssiges Metall schichtweise auf die Oberfläche aufgetragen und anschließend thermisch nachverdichtet [Sch11]. Dadurch wird ein hoher Verschleißschutz erzielt. Eine weitere Methode zur Verringerung des Oberflächenverschleißes ist das Anbringen von Verschleißbelägen, die den Kontakt zwischen Fördergut und dem zu schützenden Bauteil verhindern. So werden auf die Spiraloberfläche Kunststoffbeläge geschraubt [Zam11].

Die Oberfläche des Troges kann durch Verschleißeinlagen aus Kunststoff geschützt werden. Verschleißeinlagen aus nicht rostendem Stahl (Manganstahl) sind ebenfalls im Einsatz [App11]. 


\section{$3 \quad$ Entwicklung der modularen Spirale aus Kunststoff}

\subsection{Anforderungen}

Die Konstruktion erfolgte entsprechend der in Tabelle 1 aufgelisteten Anforderungen.

\begin{tabular}{|c|c|}
\hline Geometrie & $\begin{array}{l}\text { Gesamtlänge = } 2000 \text { mm; Modullänge = } 100 \text { mm; } \\
\text { Außendurchmesser = } 100 \text { mm; Steigung = } 100 \text { mm; } \\
\text { Wandstärke < } 6 \text { mm; förderfunktionsgerechte Geometrie }\end{array}$ \\
\hline Kinematik & $\begin{array}{l}\text { Schüttgutförderung schiebend und ziehend; } \\
\text { Geschwindigkeit = 0,15 m/s; Drehzahl = 1,5 1/s }\end{array}$ \\
\hline $\begin{array}{l}\text { Kräfte/ } \\
\text { Drehmomente }\end{array}$ & $\begin{array}{l}\text { maximale Zugkraft/ Druckkraft }=200 \mathrm{~N} \text {; } \\
\text { maximales Drehmoment }=40 \mathrm{Nm}\end{array}$ \\
\hline Schüttgut & $\begin{array}{l}\text { Stäube aus der Abgasbehandlung trocken; Gießereialtsande } \\
\text { trocken; Magnesiumchloridschuppen; Magnesiumchloridsole; } \\
\text { Kaliumchlorid feucht; Natriumchlorid feucht; Temperaturen: } 5^{\circ} \mathrm{C} \text { bis } \\
50{ }^{\circ} \mathrm{C}\end{array}$ \\
\hline $\begin{array}{l}\text { Herstellung/ } \\
\text { Montage }\end{array}$ & Herstellung durch Spritzgießen; Montage der Prototypen manuell \\
\hline
\end{tabular}

Tabelle 1: Anforderungsliste

\subsection{Gestaltung der Module}

Bei der Gestaltung der Modulgeometrie bestand die Problematik den komplexen Anforderungen gerecht zu werden (Abbildung 4).

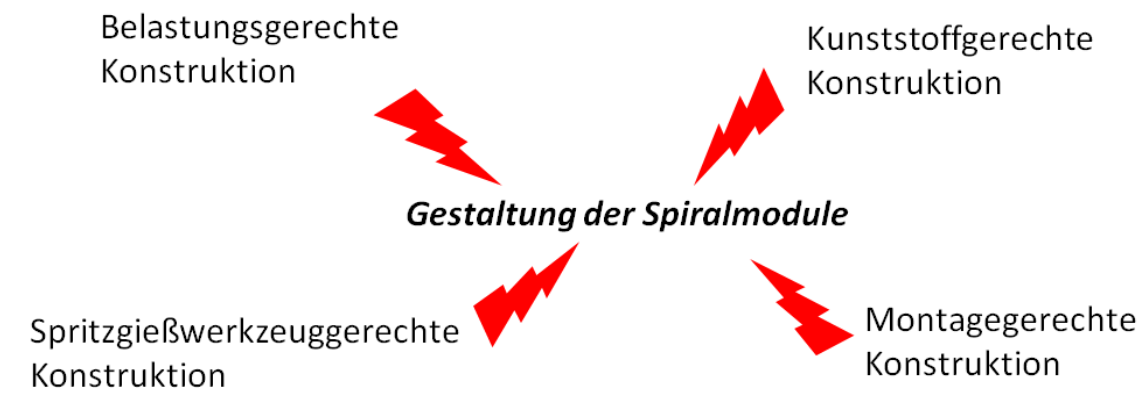

Abbildung 4: Spannungsfeld der Modulgestaltung

Die erste Stoßrichtung sah vor, die Geometrie einer Stahlspirale durch unterschiedlich angeordnete Stege hinsichtlich der Torsions- und Zugbelastung zu versteifen (Abbildung 5). Für diese Varianten wurden anschließend Verbindungsstellen gestaltet (Abbildung 6). 


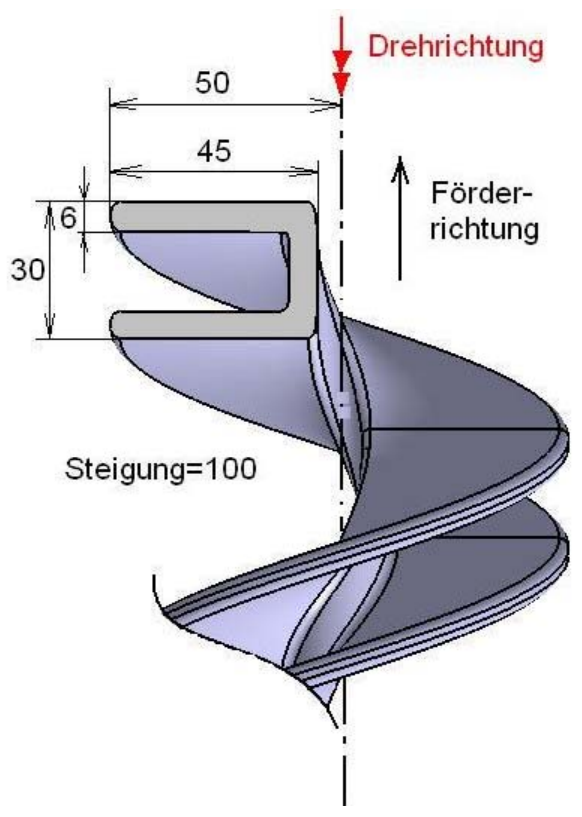

Abbildung 5: Variante 8
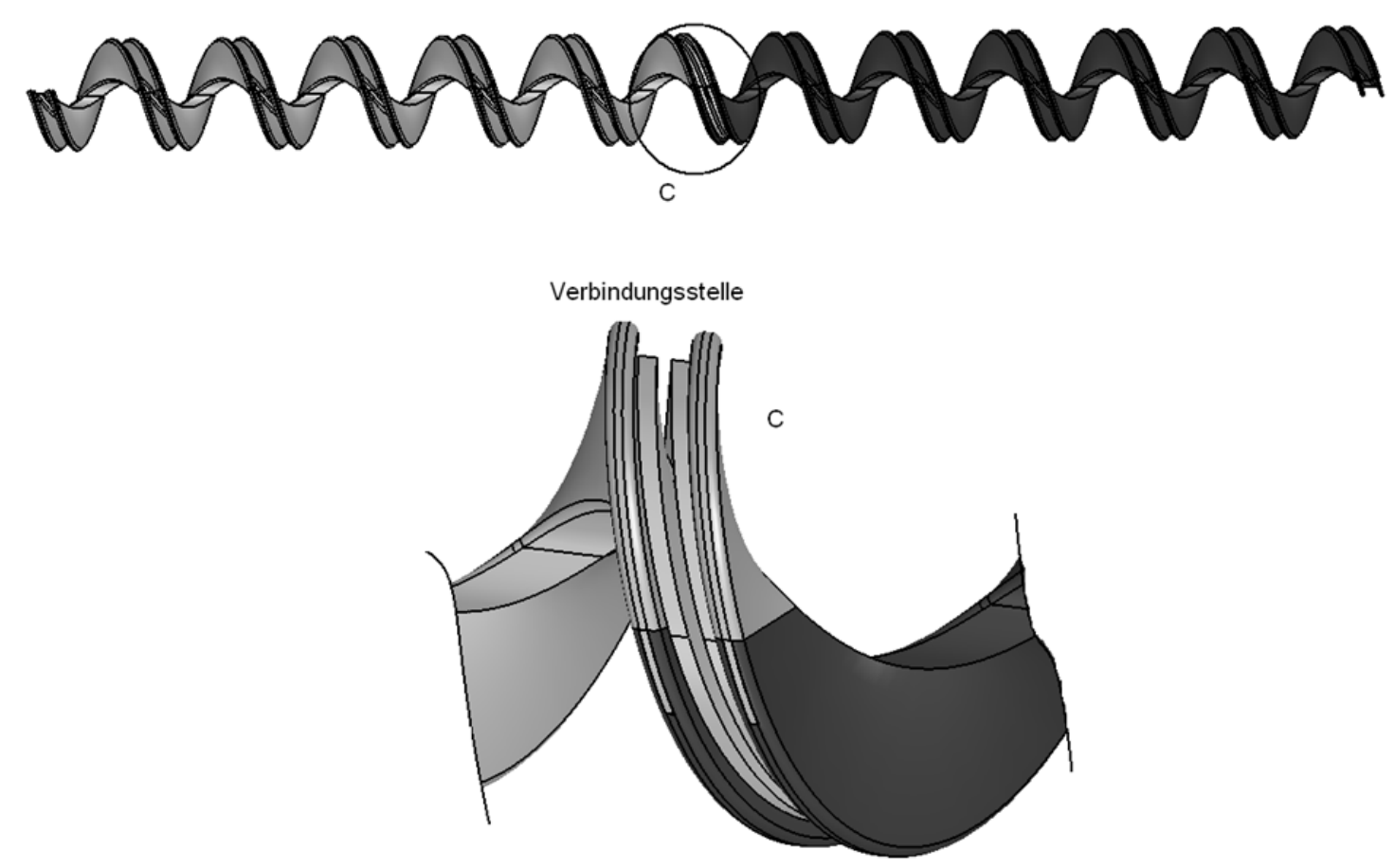

Abbildung 6: Verbindungsgestalt der Variante 8

Bei der technischen und wirtschaftlichen Bewertung der Lösungsvarianten zeigte sich jedoch schnell, dass die gewonnene Steifigkeit der Modulgeometrie durch die Verbindungsstelle stark reduziert wird. Des Weiteren sind diese Varianten nur mit komplizierten Spritzgießwerkzeugen herstellbar.

Es musste ein neuer Weg beschritten werden. Zielstellung dabei war es, die Verbindungsstelle gleichzeitig als Versteifungselement zu nutzen. Dies ist durch Rippen gelungen, die werkzeuggerecht in Entformungsrichtung orientiert sind. Ausgehend von der Mitte des Moduls ist rechtsseitig eine halbe Spiralwindung mit 
Positivstruktur und linksseitig eine halbe Spiralwindung mit Negativstruktur angeordnet (Abbildung 7). Eine Spirale beliebiger Länge kann somit aus Modulen ein und derselben Gestalt zusammengesetzt werden (Abbildung 8). Dabei greifen die Versteifungsrippen der linksseitigen Spiralwindung in die Versteifungsrippen der rechtsseitigen Spiralwindung.
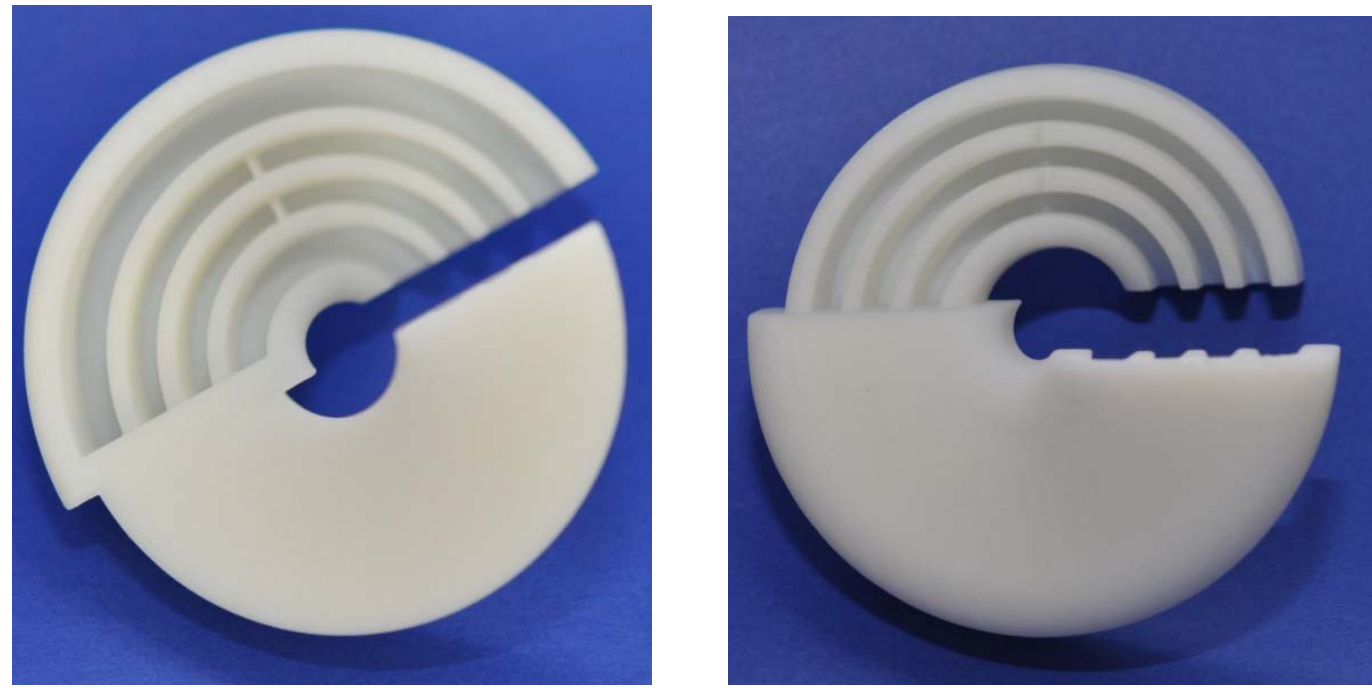

Abbildung 7: Spirale Ansicht von hinten (links); Spirale Ansicht von vorn (rechts)

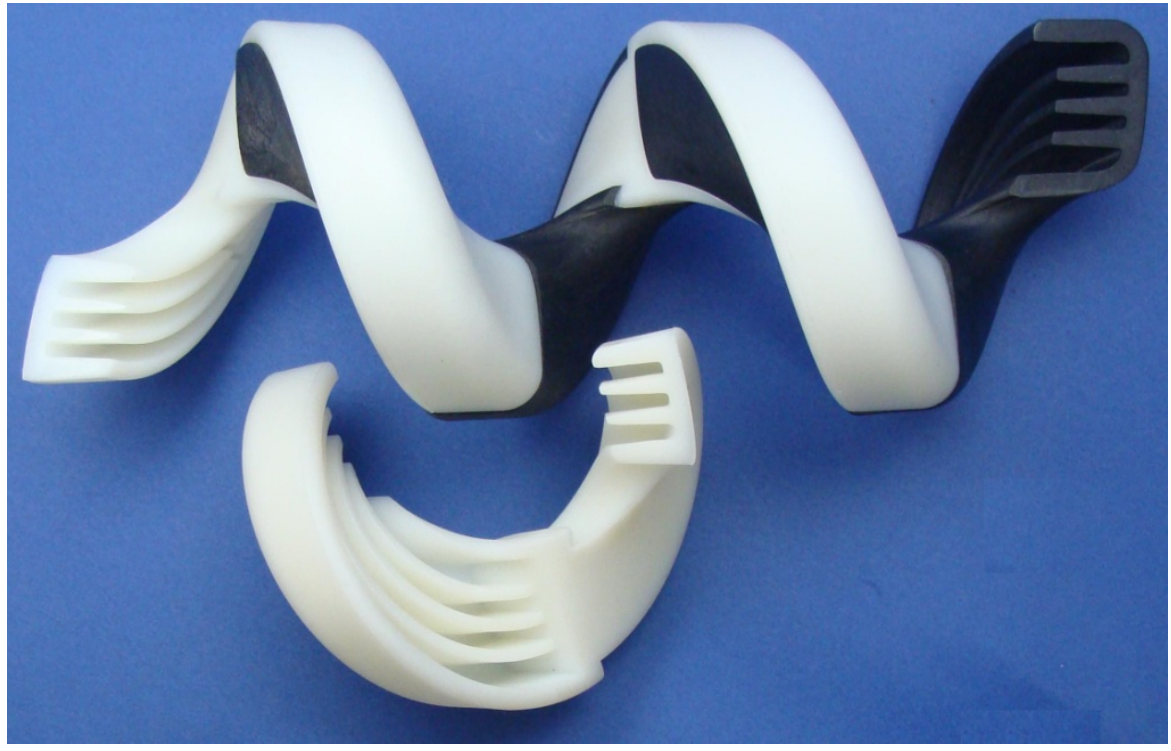

Abbildung 8: Einzelteil und vier Teile gefügt

Die dauerhafte Verbindung der Module wird durch Schmelzklebstoff oder durch Schnappverbindungen erreicht.

\subsection{Materialauswahl}

Verschiedene unverstärkte und faserverstärkte Kunststoffe wurden zur Herstellung der Module ausgewählt. Zu nennen sind in diesem Zusammenhang PP LGF60, PA6 GF30, PBT GF30, PP, PA6 und PC. Um die chemische Widerstandsfähigkeit nachzuweisen, wurden Zugstäbe aus den Kunststoffen PP LGF60, PA6 GF30 und 
PBT GF30 in den unter den Anforderungen aufgelisteten Chemikalien 14 Tage gelagert. Die mechanischen Eigenschaften von unbehandelten und von eingelagerten Proben wurden per Zugversuch ermittelt. Es konnten keine sichtbaren Änderungen und Einbußen in den mechanischen Eigenschaften durch die Wirkung der aggressiven Stoffe festgestellt werden.

\section{$4 \quad$ Versuchsergebnisse}

\subsection{Mechanische Eigenschaften der Kunststoffspirale}

Die Ermittlung mechanischer Kennwerte erfolgt mittels einer Zug- und Torsionsprüfmaschine. Je Kunststoff wurden fünf Probekörper bestehend aus 7 Modulen getestet. Die Module wurden durch Schmelzklebstoff fest verbunden. Abbildung 9 zeigt einen in die Prüfmaschine eingespannten Probekörper. Der Versuche wurde unter realitätsnahen Belastungen durchgeführt. Bei jedem Versuch wurde eine konstante Zugkraft von rund $220 \mathrm{~N}$ eingestellt (und gehalten) und anschließend die Torsionsbelastung bis zum Bruch der Proben erhöht. Diagramm 1 zeigt die Ergebnisse.

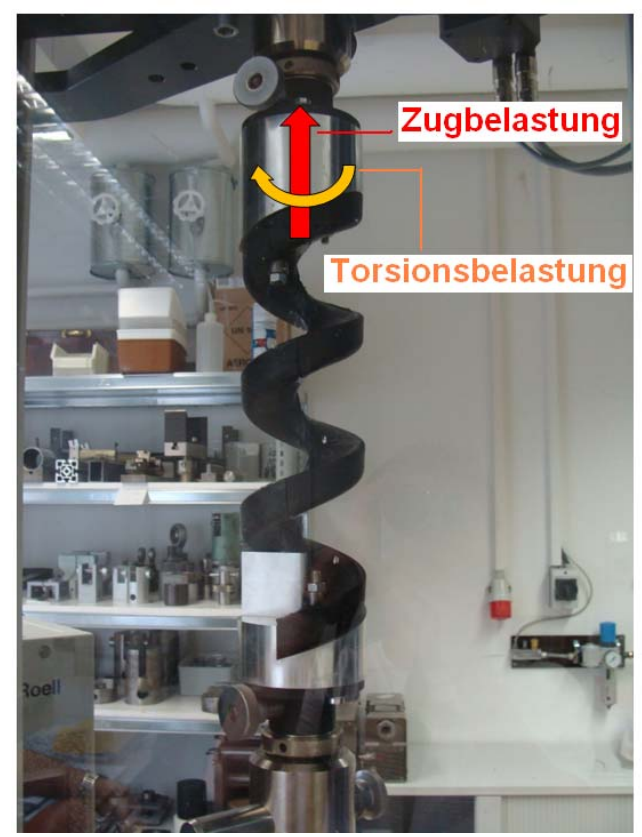

Abbildung 9: Spiralabschnitt in der Zug- und Torsionsprüfmaschine eingespannt 


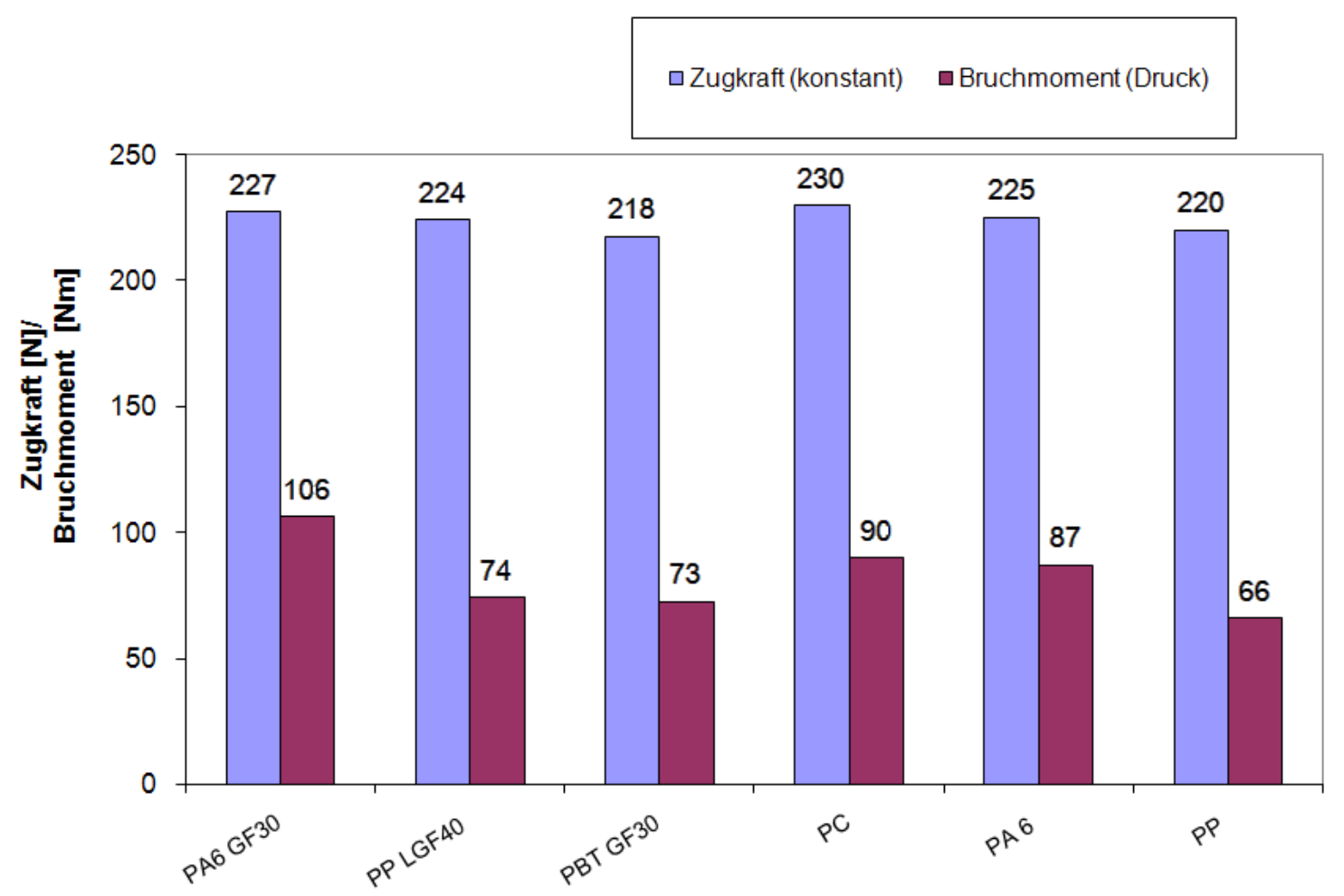

Diagramm 1: Ergebnisse der Zug- und Torsionsversuche

Das höchste Bruchmoment von $\mathrm{M}=106 \mathrm{Nm}$ bei einer konstanten Zugkraft von rund $\mathrm{Fz}=220 \mathrm{~N}$ wurde mit den Probekörpern aus PA6 GF30 erreicht. Die unverstärkten Kunststoffe PC und PA6 weisen ebenfalls einen hohen Widerstand gegen Bruchversagen auf.

\subsection{Fördereigenschaften der Kunststoffspirale}

Die Ermittlung der Fördereigenschaften der modularen Kunststoffspiralen erfolgte mit einem selbst entwickelten Prüfstand (Abbildung 10). Die Proben (Abbildung 11) wurden aus 40 Modulen zusammengesetzt und mit Schmelzklebstoff verklebt. Im Anschluss erfolgte die Verbindung zum Antriebsflansch. Getestet wurden Module aus glasfaserverstärktem und unverstärktem PA 6. Die Auswahl resultiert aus den hervorragenden mechanischen Eigenschaften, die mittels der Zug-/Torsionsversuche bestimmt wurden. 


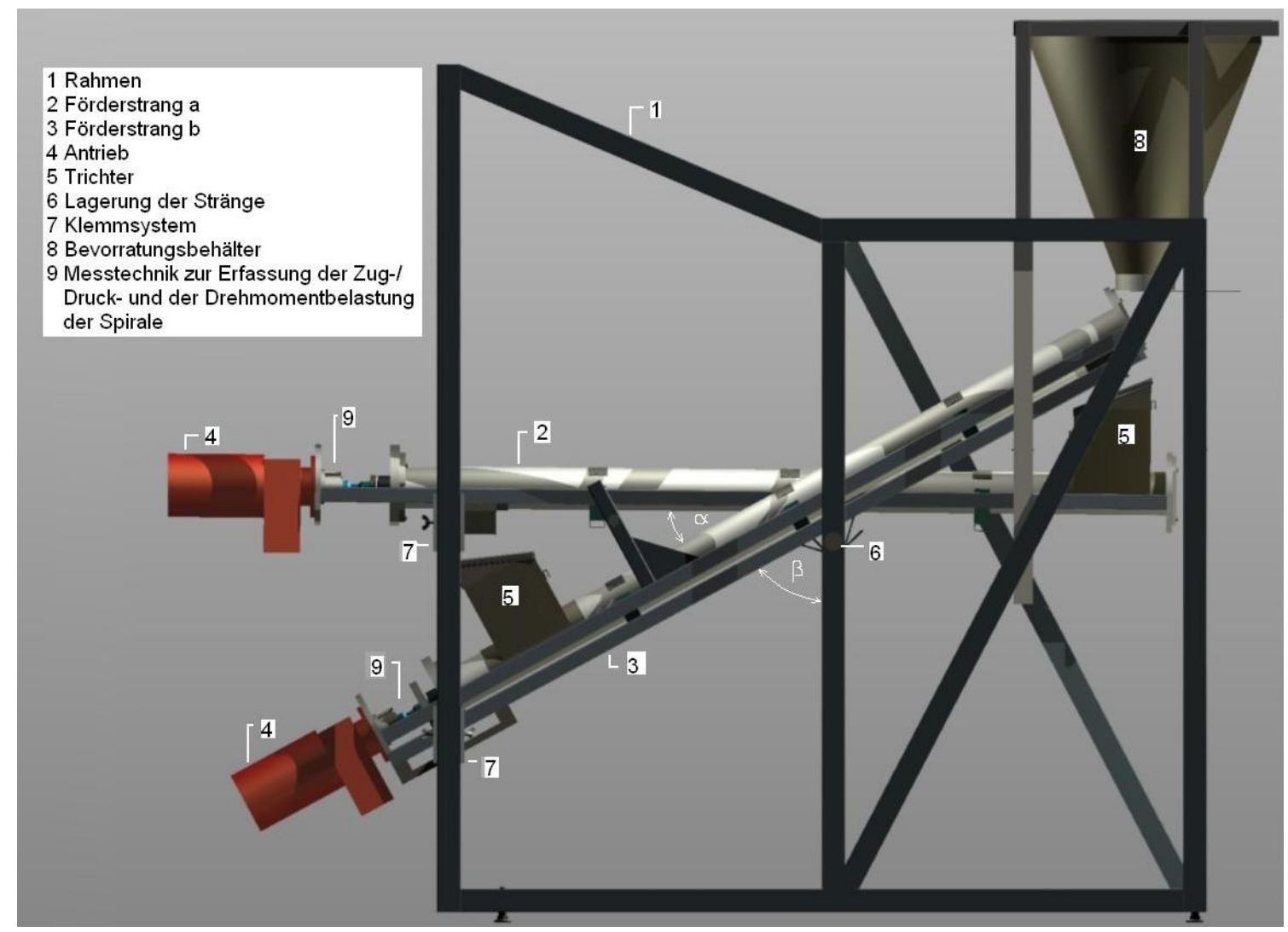

Abbildung 10: Versuchsanlage für umlaufenden Schüttguttransport

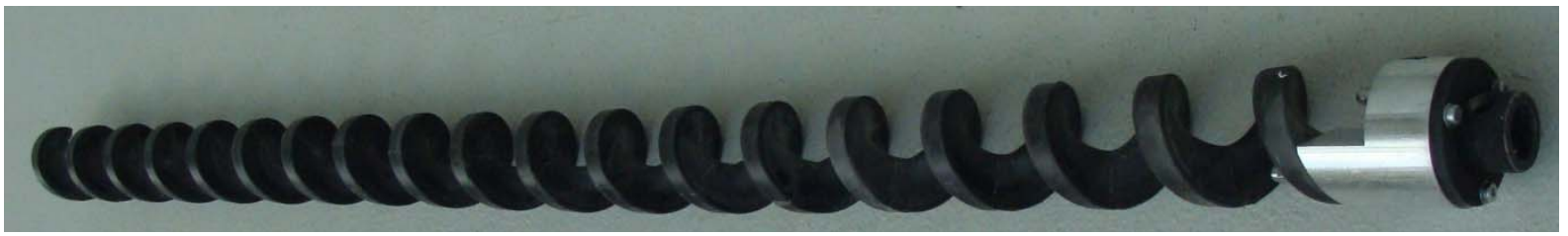

Abbildung 11: Spirale aus Kunststoffmodulen mit Antriebsflansch rechts

Zwei verschiedene Schüttgüter wurden verwendet, zum einen Sand, ein schweres, sehr abrasives Gut mit der Schüttdichte von $1,60 \mathrm{~kg} / \mathrm{dm}^{3}$ und zum anderen glasfaserverstärktes Kunststoffgranulat, ein leichtes, abrasives Gut mit der Schüttdichte $0,68 \mathrm{~kg} / \mathrm{dm}^{3}$. Diese Stoffe wurden ausgewählt um die Eigenschaften Verschleiß, Zeitfestigkeit sowie Masse- und Volumenstrom der Spiralen zu bestimmen.

Der Masse- und Volumenstrom wurde bei einer Drehzahl von 60/min und verschiedenen Anstellwinkeln $\left(0^{\circ} ; 10^{\circ} ; 2^{\circ} ; 2^{\circ}\right)$ des Rohrtroges ermittelt. Die Versuchsdurchführung erfolgte jeweils unter maximaler Befüllung des Troges mit dem Schüttgut. In dem folgenden Diagramm sind die Versuchsergebnisse dargestellt. 


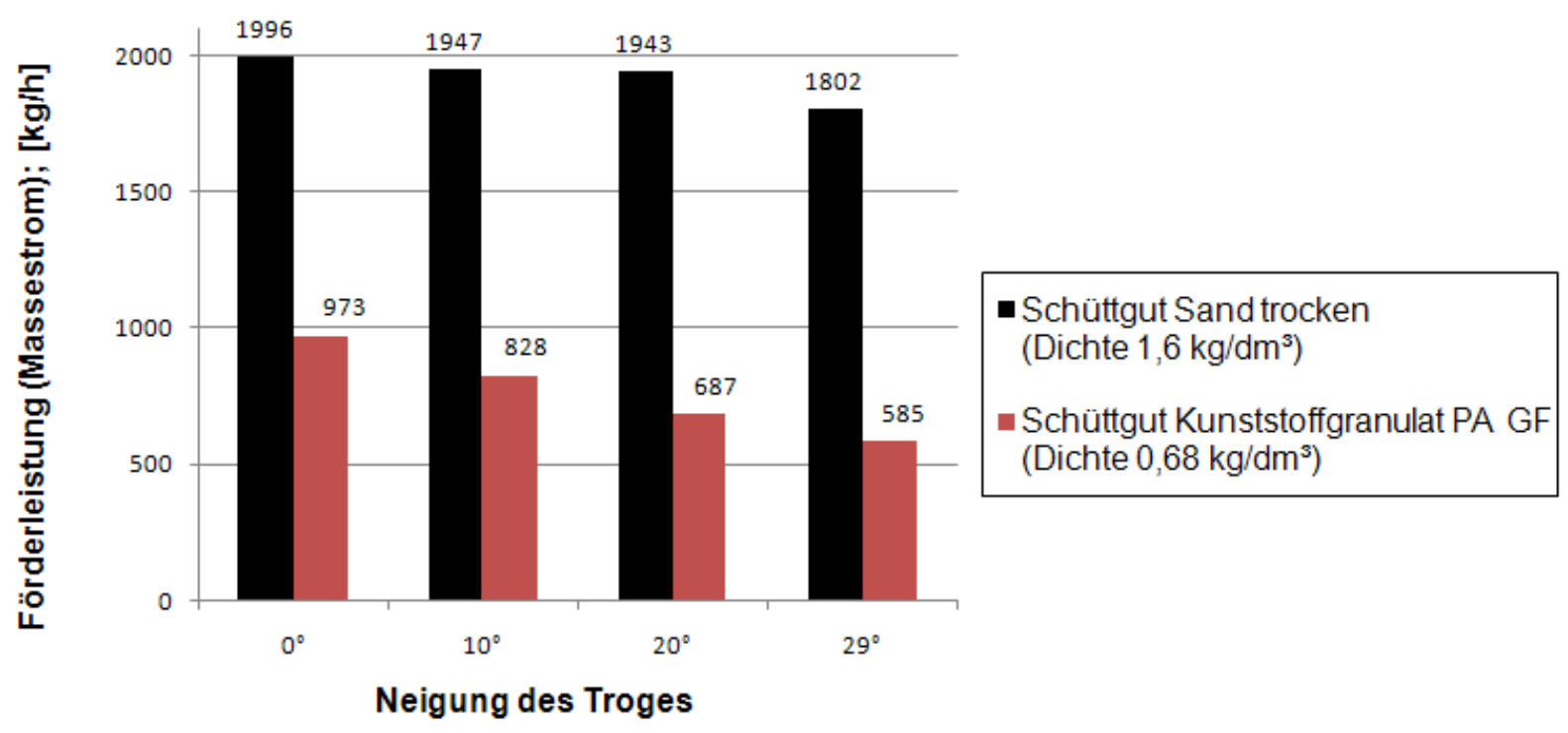

Diagramm 2: Massestrom bei einer Drehzahl von $n=60 / \mathrm{min}$

Mit dem Schüttgut Sand wird beim horizontalen Transport der maximale Massestrom von $1996 \mathrm{~kg} / \mathrm{h}$ erreicht. Beim horizontalen Transport von Kunststoffgranulat liegt der höchste Massestrom der neuen Spirale bei 973 kg/h.

\subsection{Dauerversuch}

Zwei modulare Kunststoffspiralen wurden im Dauerversuch im Prüfstand nach Abbildung 10 getestet. Die Spirale eins aus PA6 GF30 kam im Förderstrang a und Spirale zwei aus PA6 im Förderstrang b um $30^{\circ}$ geneigt zum Einsatz. Die Spirale zwei wurde mit $60 / \mathrm{min}$ betrieben. Der Volumenstrom mit dem Schüttgut Kunststoffgranulat (Schüttdichte $=0,7 \mathrm{~kg} / \mathrm{dm}^{3}$ ) betrug $\mathrm{V} / \mathrm{h}=1,4 \mathrm{~m}^{3} / \mathrm{h}$. Durch die gekreuzte Anordnung und die Übergabestellen wird die umlaufende Schüttgutförderung möglich. Bei der Versuchsdurchführung wurde an der Spirale eins ein Antriebsmoment von $M=4 \mathrm{Nm}$. Das Antriebsmomtent von Spirale zwei betrug $M=6 \mathrm{Nm}$. Nach 160 Tagen wurde der Dauerversuch abgebrochen. Bei der Inspektion der Kunststoffspiralen konnten keine Funktionseinschränkungen festgestellt werden. An der Oberfläche in Richtung Trogwand konnten Kratzer und Riefen von wenigen zehntel Millimetern festgestellt werden. Die Verschleißspuren an den Stahltrögen waren gegenüber dem Verschleiß an den Spiralen stärker ausgeprägt.

\section{Literatur}

[App11] Applied Chemicals Handels-G.m.b.H: Spiralförderer, http://www.acat.com/de/produkte/machine-tech/667, 02-2011

[Ste11] Steiner GmbH \& Co KG: Starre Spiralen, http://www.steiner-spiralen.de, 02-2011

[VDI93] VDI 2330: Schneckenförderer, 1993 
[DIN83] DIN 15262: Schneckenförderer für Schüttgut, Berechnungsgrundsätze, 1983

[Sch11] Schrage GmbH: Thermisches Spritzen, http://www.schragegmbh.de/metallsp_schwtech/prod_thermisches_spritzen.php3, 02-2011

[Zam11] ZAM Kety Sp. Z o.o.: Spiralbeläge für Klassierer, http://www.zam.com.pl/zam/ind_de.htm, 02-2011 